\title{
Rheumatoid arthritis-associated polymorphisms are not protective against Alzheimer's disease
}

\author{
Christopher R Simmons ${ }^{1}$, Fanggeng Zou², Steven G Younkin ${ }^{2}$ and Steven Estus ${ }^{1 *}$
}

\begin{abstract}
Background: Rheumatoid arthritis (RA) and Alzheimer's disease (AD) are inversely associated. To test the hypothesis that genetic elements associated with increased RA risk are associated with decreased AD risk, we evaluated RA genetic risk factors recently identified in genome-wide association studies (GWAS) for their association with $\mathrm{AD}$ in a two-stage, case-control analysis.
\end{abstract}

Results: In our Stage 1 analysis of $\sim 800$ AD and 1,200 non-AD individuals, three of seventeen RA-associated SNPS were nominally associated with AD ( $p$ <.05) with one SNP, rs2837960, retaining significance after correction for multiple testing $(p=0.03)$. The rs2837960_G (minor) allele, which is associated with increased RA risk, was associated with increased AD risk. Analysis of these three SNPs in a Stage 2 population, consisting of $\sim 1,100 \mathrm{AD}$ and 2,600 non-AD individuals, did not confirm their association with AD. Analysis of Stage 1 and 2 combined suggested that rs2837960 shows a trend for association with AD. When the Stage 2 population was age-matched for the Stage 1 population, rs2837960 exhibited a non-significant trend with AD. Combined analysis of Stage 1 and the age-matched Stage 2 subset showed a significant association of rs2837960 with AD ( $p=0.002$, OR 1.24) that retained significance following correction for age, sex and APOE ( $p=0.02, O R=1.20)$. Rs2837960 is near BACE2, which encodes an aspartic protease capable of processing the AD-associated amyloid precursor protein. Testing for an association between rs2837960 and the expression of BACE2 isoforms in human brain, we observed a trend between rs2837960 and the total expression of BACE2 and the expression of a BACE2 transcript lacking exon 7 ( $p=0.07$ and 0.10 , respectively).

Conclusions: RA-associated SNPs are generally not associated with AD. Moreover, rs2837960_G is associated with increased risk of both RA and, in individuals less than 80 years of age, with AD. Overall, these results contest the hypothesis that genetic variants associated with RA confer protection against AD. Further investigation of rs2837960 is necessary to elucidate the mechanism by which rs2837960 contributes to both AD and RA risk, likely via modulation of BACE2 expression.

\section{Background}

There is a long-standing, inverse relationship between the prevalence of Alzheimer's disease (AD) and of rheumatoid arthritis (RA). Jenkinson and colleagues first described the decreased prevalence of RA in patients suffering from senile dementia of the Alzheimer's type as compared to cognitively intact individuals [1]. Further retrospective studies of clinical and autopsy data revealed that patients with RA exhibit a reduced prevalence of $\mathrm{AD}[2]$. A study by Myllykangas-Luosujarvi and

\footnotetext{
* Correspondence: steve.estus@uky.edu

'Department of Physiology, Sanders-Brown Center on Aging, University of Kentucky, Lexington, KY, USA

Full list of author information is available at the end of the article
}

colleagues evaluating AD pathology in patients with and without RA revealed that AD-associated neuropathology occurred four times less often in patients with RA as compared to the general population [3].

The basis of this inverse relationship is unclear but may include both genetic and environmental factors. RA and $\mathrm{AD}$ each have a strong genetic component, i.e., $50 \%$ of RA risk and $60 \%$ of $\mathrm{AD}$ risk is attributable to genetic factors, supporting the original hypothesis of Jenkinson and colleagues that genetics might explain the relationship between AD and RA [4,5]. Alternatively, antiinflammatory medications used therapeutically for the treatment of RA could decrease AD risk by reducing $\mathrm{AD}$-associated inflammation or via other mechanisms, .

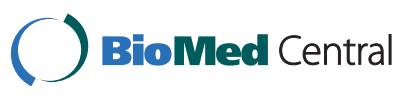


e.g., modulation of APP processing [6,7]. Supporting this possibility, an initial double-blind, placebo-controlled study by Rogers et al. provided evidence that indomethacin slowed cognitive decline in patients with $\mathrm{AD}$ relative to placebo [8]. These findings were further supported by Breitner and colleagues who found that multiple anti-inflammatory medications slow disease progression and delay disease onset [9]. However, there has been little success replicating these findings in larger, randomized clinical trials [10-13]. Hence, whether anti-inflammatory agents delay the onset of $\mathrm{AD}$ remains unclear.

The recent advent of RA genome wide association studies (GWAS) has identified single nucleotide polymorphisms (SNP)s associated with RA that provide a foundation for evaluating the initial hypothesis of Jenkinson $e$ al. that genetic variants that increase the risk of RA also decrease the risk of AD. To this end, we tested whether seventeen RA-associated SNPs with genome-wide significance were associated with $\mathrm{AD}$ in a two-stage analysis using separate AD case-control populations. We found that none of the seventeen alleles associated with increased RA risk were also associated with reduced AD risk. Rather, we found three RA-associated SNPs that were nominally associated with AD $(p<0.05)$. One of these SNPs, rs2837960, was found to be significantly associated with $\mathrm{AD}$ in a combined analysis of our Stage 1 and Stage 2 populations when the Stage 2 population was restricted to individuals of similar age as Stage 1. The gene closest to rs 2837960 is BACE2, the product of which has been implicated in amyloid protein precursor (APP) processing $[14,15]$. When we evaluated the expression of $B A C E 2$ isoforms as a function of rs2837960, we found a trend for $B A C E 2$ expression with rs2837960. In summary, genetic variants that increase RA risk do not decrease AD risk. The inverse relationship between RA and AD may thus be better explained by environmental factors such as the use of anti-inflammatory medications. Further functional investigation of rs2837960 is needed to elucidate the mechanism by which this SNP may modulate AD and RA.

\section{Results}

RA-associated SNPs are generally not associated with AD To evaluate whether RA-associated SNPs are also associated with AD, we began by identifying SNPs that are robustly associated with RA risk and then evaluated these SNPs for their association with AD in an exploratory Stage 1 case-control population of $\sim 800$ AD and $\sim 1200$ non-AD individuals. Contrary to the hypothesis that alleles associated with increased RA risk are also associated with reduced AD risk, only three of the seventeen RA-associated SNPs in our Stage 1 study were nominally significant for association with AD $(\mathrm{p}<0.05$, Table 1$)$. Moreover, for each these SNPs, the allele associated with increased RA risk was also associated with increased AD risk, further refuting the hypothesis that genetics underlies the inverse epidemiologic relationship between RA and $\mathrm{AD}$ prevalence. The $\mathrm{AD}$-associated SNPs are located in or near the genes BACE2 (rs2837960; $\mathrm{p}=0.002$, OR = 1.29), TRAF1/C5 (rs3761847; $\mathrm{p}=0.006, \mathrm{OR}=1.19)$ and SALL3 (rs2002842; $\mathrm{p}=0.04$, OR $=1.15$ ). When a Bonferroni correction for multiple testing was applied to minimize false-positive associations between RA-associated SNPs and AD, only rs2837960 exhibited a significant association with AD $(\mathrm{p}=0.03)$. Furthermore, rs 2837960 remained significantly associated with $\mathrm{AD}$ after correcting for age, sex and $A P O E$ genotype per logistic regression $(\mathrm{p}=0.012 \mathrm{OR}=1.26$, Table 2). Rs3761847 remained significant following correction of Stage 1 analysis for covariates $(\mathrm{p}=0.007 \mathrm{OR}=1.21)$.

\section{Rs2837960 is significantly associated with AD in individuals}

The three RA- and potentially AD-associated SNPs from Stage 1 were evaluated further for their association with AD in a Stage 2 case-control series that consisted of 2677 non-AD and 1102 AD subjects. Contrary to our initial results, these SNPs exhibited no association with AD in our Stage 2 population (Table 3). Correction for age, sex and APOE genotype had marginal, non-significant effects on the association between these three SNPs and AD. Our Stage 1 and Stage 2 populations were combined with the intention of clarifying the overall association between these three SNPs and AD. Analysis of this combined population, consisting of 3949 non-AD and 1965 AD subjects, suggested that rs 2837960 is significantly associated with $\mathrm{AD}(\mathrm{p}=0.04, \mathrm{OR}=1.11$, Table 4$)$. When these results were corrected for age, sex and $A P O E$ genotype this association between $\mathrm{rs} 2837960$ and AD showed only a trend $(\mathrm{p}=0.14)$.

Due to the large discrepancy in average age between our Stage 1 and Stage 2 populations, and the possibility that the impact of genetic risk factors may decline with age, we next evaluated a subset of Stage 2 individuals that had an age of AD diagnosis between 60 and 80 years of age along with non-AD individuals with the same age range [16]. Thus, our age parameters and average population ages for Stage 2 mimicked those of Stage 1. This effort resulted in a sample population that consisted of $186 \mathrm{AD}$ (average age 73) and 912 non-AD individuals (average age of 74). This population showed a trend in the OR of rs2837960 that was consistent with that of the Stage 1 analysis (Table 5), i.e., the minor rs2837960_G allele appeared to impart an increased, although non-significant, risk of AD. The SNPs rs3761847 and rs2002842, that were associated with AD in Stage 1, failed to show an association with AD in Stage 2. We note that there was significant overlap in 
Table 1 Stage 1 Analysis of RA-SNPs Association with AD

\begin{tabular}{|c|c|c|c|c|c|c|c|}
\hline CHR & SNP & Gene & RA OR & RA P-value & AD OR $[96 \% \mathrm{Cl}]$ & AD P-value & BF p-value \\
\hline 21 & rs2837960 & BACE2 & 1.05 & $2 \times 10^{-6}$ & $1.29[1.10-1.52]$ & 0.002 & 0.03 \\
\hline 9 & rs3761847 & TRAF1, C5 & 1.32 & $4 \times 10^{-14}$ & 1.19 [1.05-1.35] & 0.006 & 0.11 \\
\hline 18 & rs2002842 & SALL3 & 1.61 & $6 \times 10^{-6}$ & 1.15 [1.01-1.31] & 0.04 & 0.65 \\
\hline 9 & rs881375 & TRAF1, C5 & NR & $4 \times 10^{-8}$ & $1.11[0.97-1.26]$ & 0.12 & \\
\hline 6 & rs660895 & HLA-DRB1 & 3.62 & $1 \times 10^{-108}$ & 0.93 [0.80-1.09] & 0.39 & \\
\hline 12 & rs3184504 & $\mathrm{SH} 2 \mathrm{~B} 3$ & 0.92 & $6 \times 10^{-6}$ & 0.95 [0.84-1.08] & 0.41 & \\
\hline 8 & rs2736340 & BLK & 1.19 & $6 \times 10^{-9}$ & $0.95[0.82-1.10]$ & 0.47 & \\
\hline 2 & rs13031237 & REL & 1.13 & $8 \times 10^{-7}$ & 1.04 [0.92-1.19] & 0.52 & \\
\hline 6 & rs6457617 & HLA-E & 2.36 & $5 \times 10^{-75}$ & $1.03[0.91-1.17]$ & 0.61 & \\
\hline 4 & rs13119723 & IL2, IL21 & 1.12 & $7 \times 10^{-7}$ & $0.96[0.80-1.14]$ & 0.62 & \\
\hline 2 & rs13017599 & REL & 1.21 & $2 \times 10^{-12}$ & 1.03 [0.90-1.17] & 0.67 & \\
\hline 9 & rs951005 & CCL21 & 0.81 & $4 \times 10^{-10}$ & $1.03[0.87-1.22]$ & 0.73 & \\
\hline 6 & rs6910071 & HLA-DRB1 & 2.88 & $1 \times 10^{-299}$ & $0.98[0.84-1.14]$ & 0.75 & \\
\hline 1 & rs2476601 & PTPN22 & 1.94 & $9 \times 10^{-74}$ & $1.03[0.84-1.27]$ & 0.77 & \\
\hline 7 & rs10488631 & IRF5 & 1.19 & $4 \times 10^{-11}$ & $0.99[0.81-1.20]$ & 0.91 & \\
\hline 2 & rs231735 & CTLA4 & 0.83 & $6 \times 10^{-9}$ & 1.01 [0.89-1.14] & 0.91 & \\
\hline 1 & rs3890745 & TNFRSF14 & 0.88 & $4 \times 10^{-6}$ & 0.99 [0.87-1.13] & 0.93 & \\
\hline
\end{tabular}

Seventeen SNPs exhibiting genome-wide significant associations with RA were tested for their association with AD risk using allelic models in a Mayo Clinic AD GWAS series of 843 AD and 1264 non-AD individuals. Three of the seventeen RA-associated SNPs were nominally associated with AD ( $p<0.05$ ). Multiple testing was addressed by applying a Bonferroni (BF) correction for the number of tests performed. The only SNP that retained significance after BF correction was rs2837960 ( $p=0.03)$. NR = value not reported in initial study.

the 95\% confidence intervals between Stage 1 and Stage 2 for all three of these SNPs. Analysis of the combined data from Stage 1 and the Stage 2 subset revealed that only rs2837960 maintained a significant association with

Table 2 Stage 1 Analysis of RA-SNP's Association with AD Corrected for Age, Sex and APOE Genotype

\begin{tabular}{|c|c|c|c|c|c|}
\hline $\mathrm{CHR}$ & SNP & OR & L95 & U95 & $\mathbf{P}$ \\
\hline 9 & rs3761847 & 1.211 & 1.054 & 1.392 & 0.007 \\
\hline 21 & rs2837960 & 1.256 & 1.051 & 1.502 & 0.012 \\
\hline 18 & rs2002842 & 1.147 & 0.995 & 1.323 & 0.059 \\
\hline 9 & rs881375 & 1.125 & 0.973 & 1.300 & 0.111 \\
\hline 6 & rs660895 & 0.903 & 0.759 & 1.076 & 0.254 \\
\hline 8 & rs2736340 & 0.920 & 0.786 & 1.077 & 0.298 \\
\hline 2 & rs13031237 & 1.067 & 0.926 & 1.230 & 0.372 \\
\hline 6 & rs6457617 & 1.056 & 0.921 & 1.211 & 0.435 \\
\hline 2 & rs13017599 & 1.055 & 0.914 & 1.217 & 0.467 \\
\hline 9 & rs951005 & 1.060 & 0.880 & 1.278 & 0.539 \\
\hline 6 & rs6910071 & 0.949 & 0.797 & 1.130 & 0.558 \\
\hline 2 & rs231735 & 1.027 & 0.895 & 1.178 & 0.705 \\
\hline 7 & rs10488631 & 0.961 & 0.774 & 1.193 & 0.717 \\
\hline 4 & rs13119723 & 0.969 & 0.800 & 1.174 & 0.746 \\
\hline 1 & rs3890745 & 1.022 & 0.882 & 1.186 & 0.770 \\
\hline 1 & rs2476601 & 1.018 & 0.811 & 1.278 & 0.880 \\
\hline 12 & rs3184504 & 0.998 & 0.869 & 1.146 & 0.976 \\
\hline
\end{tabular}

Using logistic regression, SNP-AD associations were corrected for the potentia effects of age, sex and APOE genotype (all of which were independent predictors of AD in our Stage 1 analysis). The minor alleles of both rs2837960 and rs3761847 were significantly associated with increased risk of $A D(p<$ 0.05 ) while the minor allele of rs2002842 exhibited only a trend toward an increased risk of $A D$.
AD $(p=0.002$, OR $=1.27$, Table 6$)$. This association between rs2837960 and AD remained significant following correction for the covariates age, sex and APOE ( $p$ $=0.02$, OR $=1.20)$. Thus, when the Stage 1 and Stage 2 populations were matched for age, rs2837960 was significantly associated with AD risk.

\section{In silico analyses suggest that rs2837960 is associated with BACE2 expression and splicing}

To gain insights into the possible actions of rs2837960, we first identified genes within the vicinity of rs2837960 by using HapMap [17]. This analysis found that rs2837960 resides within a haplotypic block that includes the promoter region and 5'-UTR of BACE2 (Figure 1A). Due to the presence of distinct proximal and distal $B A C E 2$ promoters, and the potential contribution of upstream regulatory elements to $B A C E 2$ expression, we considered the possibility that rs3846662 may affect $B A C E 2$ expression [18-20]. We then queried whether there was an association between rs 2837960 and $B A C E 2$ expression in human brain by using the SNPexpress database, which includes data from 93 human brain samples [21]. This analysis revealed a trend towards increased $B A C E 2$ expression in rs2837960 minor allele carriers $(\mathrm{p}=0.08$, Figure $1 B)$. Since these data suggest that rs2837960 may modulate $B A C E 2$ expression and since BACE2 function in turn may modulate $\mathrm{AD}$ and cognition, we hypothesized that rs2837960 modulates $\mathrm{AD}$ risk by altering $B A C E 2$ expression in the human brain [22-24]. 
Table 3 Stage 2 Analysis of Top RA-SNPs Associated with AD

\begin{tabular}{cccccccccc}
\hline & & \multicolumn{3}{c}{ Uncorrected Logistic Regression } & \multicolumn{4}{c}{ Regression Corrected for Age, Sex and APOE } \\
CHR & SNP & OR & L95 & U95 & P & OR & L95 & U95 & P \\
\hline $\mathbf{2 1}$ & rs2837960 & 1.011 & 0.887 & 1.153 & 0.865 & 1.000 & 0.867 & 1.154 & 0.997 \\
$\mathbf{1 8}$ & rs2002842 & 0.992 & 0.896 & 1.099 & 0.880 & 0.990 & 0.886 & 1.106 & 0.857 \\
$\mathbf{9}$ & rs3761847 & 0.921 & 0.832 & 1.019 & 0.112 & 0.905 & 0.809 & 1.012 & 0.080 \\
\hline
\end{tabular}

No RA-associated SNP that exhibited an association with AD in Stage 1 was found to exhibit an association with AD in our Stage 2 population. This remained true when data were analyzed and corrected for covariates including age, sex and APOE genotype of Stage 2 individuals, who on average were significantly older than Stage 1 individuals ( $p<0.001$, Students t-test).

\section{Relationship between rs2837960 and BACE2 isoforms in the human brain}

To pursue this hypothesis, we sought to directly quantify BACE2 isoforms as a function of rs2837960 in a series of human brain samples. We began by confirming the presence of previously identified $B A C E 2$ isoforms that lack exons seven or eight (BACE2d7 and $B A C E 2 d 8$, respectively) as well as the full-length, nine-exon $B A C E 2$ transcript $\left(B A C E 2 \_F L\right)$. We also identified a novel isoform lacking both exons 7 and 8 (BACE2d7/8) that was confirmed by direct sequencing (Figure 2). Considering the biological relevance of these isoforms, we note that the loss of exon 7 is predicted to result in an in-frame deletion of 50 amino acids, resulting in a $50.3 \mathrm{kDa}$ peptide. Moreover, the BACE2 protein found in human brain appears to correspond to the $B A C E 2 d 7$ isoform based upon its size and pattern of epitope reactivity [14]. In contrast, the loss of exon 8 or exons 7-8 results in a frameshift and prematurely truncated BACE2 proteins of $43.0 \mathrm{kDa}$ and $37.5 \mathrm{kDa}$, respectively. Hence, we chose to use real-time PCR to quantify $B A C E 2$ tot and $B A C E 2 d 7$ since they represent the expression of total BACE2 and functional BACE2, respectively. A total of 53 brain cDNA samples were analyzed for genotypic associations between rs2837960 and expression of $B A C E 2$ tot and BACEd7. This effort revealed a trend between rs2837960 and the expression of BACE2tot (p $=0.07)$ and $B A C E 2 d 7(\mathrm{p}=0.10$, Figure 3$)$. It is noteworthy that the trend toward greater expression of $B A C E 2$ tot and BACE2d7 in rs2837960 minor allele carriers in our results is similar to the results from SNPExpress.

Table 4 Combined Stage 1 and Stage 2 Analysis of Top RA-SNPs for Association with AD

\begin{tabular}{|c|c|c|c|c|c|c|c|c|c|}
\hline \multicolumn{6}{|c|}{$\begin{array}{l}\text { Regression Corrected for Age, Sex and } \\
\text { APOE }\end{array}$} & \multicolumn{4}{|c|}{$\begin{array}{l}\text { Uncorrected Logistic } \\
\text { Regression }\end{array}$} \\
\hline $\mathrm{CHR}$ & SNP & OR & L95 & U95 & $\mathbf{P}$ & OR & L95 & U95 & $\mathbf{P}$ \\
\hline 21 & rs2837960 & 1.113 & 1.007 & 1.230 & 0.037 & 1.087 & 0.974 & 1.212 & 0.138 \\
\hline 18 & rs2002842 & 1.031 & 0.952 & 1.116 & 0.460 & 1.017 & 0.933 & 1.110 & 0.699 \\
\hline 9 & rs3761847 & 1.011 & 0.934 & 1.093 & 0.793 & 1.002 & 0.920 & 1.091 & 0.965 \\
\hline
\end{tabular}

\section{Discussion}

The primary finding of this investigation is that the majority of seventeen SNPs that exhibit a genome-wide significant association with RA are not associated with AD. Furthermore, the minor allele of rs2837960, which was found to be significantly associated with AD risk after combined analysis of Stage 1 and age-matched Stage 2 data, was associated with an increased risk of both RA and AD. Hence, these results contest the hypothesis that genetics underlie the inverse relationship between RA and $\mathrm{AD}$, i.e. that alleles associated with an increased risk of RA are protective against AD. A secondary finding is that we have pursued the role of rs2837960 in its possible regulation of the nearby $B A C E 2$ gene. We report the presence of multiple $B A C E 2$ isoforms in human brain and that rs2837960 shows a trend for association with BACE2tot and $B A C E 2 d 7$, which represent total BACE2 and functional BACE2, respectively [14]. In summary, the genetic underpinnings of RA have negligible overlap with AD with the exception of rs2837960, which is associated with both RA and AD, possibly through its effects on $B A C E 2$ expression.

RA and AD each have a strong genetic component that accounts for approximately $50 \%$ and $60 \%$ of their risk, respectively $[4,5]$. The remainder of $\mathrm{RA}$ and $\mathrm{AD}$ risk is likely derived from environmental influences. The vast majority of RA-associated SNPs implicate gene products involved in immune system processes. Chronic inflammation of the brain is a common feature of $\mathrm{AD}$ pathology, raising the possibility that RA-associated SNPs that influence immune system function could influence AD risk [25-27]. It is well established that some of the most strongly AD-associated genes, including CLU, CR1, TNF and CCR2, exhibit ontological association with immune system processes [28-36]. Hence, the impetus for pursuing genetic overlap between RA and $\mathrm{AD}$ is greater than that provided by their epidemiologic relationship alone. However, our results indicate that RA-associated SNPs, which pertain largely to gene products involved in immune system processes, are not associated with AD.

There are several possible interpretations of our primary findings. The lack of overlap between RA-associated SNPs 
Table 5 Analysis of RA-SNP's Association with AD in Stage 2, Age-Matched to Stage 1

\begin{tabular}{cccccccccc}
\hline & \multicolumn{3}{c}{ Uncorrected Logistic Regression } & \multicolumn{4}{c}{ Regression Corrected for Age, Sex and APOE } \\
CHR & SNP & OR & L95 & U95 & P & OR & L95 & U95 & P \\
\hline $\mathbf{2 1}$ & rs2837960 & 1.218 & 0.922 & 1.609 & 0.165 & 1.225 & 0.903 & 1.664 & 0.192 \\
$\mathbf{1 8}$ & rs2002842 & 1.002 & 0.802 & 1.253 & 0.983 & 1.013 & 0.794 & 1.292 & 0.920 \\
$\mathbf{9}$ & rs3761847 & 0.897 & 0.723 & 1.113 & 0.324 & 0.878 & 0.694 & 1.110 & 0.278 \\
\hline
\end{tabular}

The three RA-associated SNPs suggesting association with $A D$ were tested further for their association with $A D$ risk by using logistic in a series of 912 non-AD and $186 \mathrm{AD}$ individuals whose ages more closely resembled those of Stage 1 individuals. Although significant associations were not observed between these SNPs and $A D$, the results for each SNP were not significantly different from those obtained in Stage 1.

and $\mathrm{AD}$ could be due in part to the tissue-specific expression of DNA and RNA binding proteins required to interact with these SNPs to manifest effects on gene expression [37]. However, if any of the seventeen RA-associated SNPs included in this study are capable of modulating peripheral immune system activity, either alone or in combination with each other, then it is probable that their peripheral effects on the immune system would indirectly affect immune system activity within the CNS. Evidence supporting the ability of peripheral inflammation to modulate CNS inflammation has been reported previously [38]. Therefore, if RA-associated SNPs are only functional in the periphery then their effects on immune system function and inflammation should manifest in the CNS, even if the same SNPs do not modulate endogenous immune system function within the brain.

What is yet unclear is whether RA-associated alleles actually propagate inflammation and, if so, why they would not be expected to increase, rather than decrease, AD risk. In fact, the results of our study suggest that alleles that increase RA risk may likewise increase AD risk, i.e., rs2738960_G increases risk of both RA and AD, while rs3761847_G and rs2002842_A show a similar trend. If these observations are replicated in future studies, alleles that are pro-inflammatory may emerge as risk factors for both RA and AD. More explicitly, considering the role of genetics and environment in RA and $A D$, these results suggest that RA genetics alone may enhance rather than reduce AD risk. Hence, the inverse epidemiologic relationship between RA and AD is likely explained by an environmental RA-associated influence. In this regard, McGeer et al. postulated that the reduced prevalence of $\mathrm{AD}$ in RA patients is related to the use of anti-inflammatory drugs for the treatment of RA [2].
Multiple studies of anti-inflammatory agents have since been performed to test for their ability to modify $A D$ risk and cognitive decline in $\mathrm{AD}$ patients, yielding mixed results [8,39-42]. To some extent, variability in study outcome may be explained by the additional ability of a subset of anti-inflammatory medications to reduce production of the neurotoxic $A \beta_{1-42}$ peptide [6]. Further investigation is required to clarify the functional genetics of RA- and AD-associated SNPs and the role of anti-inflammatory medications in AD.

In pursuit of the functional genetics of rs2837960, which is associated with an increased risk of RA and $\mathrm{AD}$, we investigated its association with the expression of BACE2 isoforms in human brain [43]. Thus, our secondary finding is that the minor allele of rs 2837960 showed a strong trend for association with increased expression of $B A C E 2$ tot and $B A C E 2 d 7$, the latter of which may represent the majority of functional BACE2 in human brain [14]. BACE2 encodes a transmembrane aspartic protease and is $\sim 75 \%$ homologous with $B A C E 1$ with regard to amino acid sequence [20]. Although the function of BACE2 is disputed, it appears to possess both $\beta$-secretase and $\alpha$-secretase-like activities [15]. Data obtained from the study of $B A C E 1 / B A C E 2$ doubleknockout mice suggest that $B A C E 2$ expressed in glia contributes significantly to $A \beta$ production [44]. This glial-specific expression is likely due to the more distal of the two distinct $B A C E 2$ promoters, neither of which share similarity with the $B A C E 1$ promoter [18-20].

Several factors are consistent with the possibility that rs2837960, or SNPs in tight linkage with rs2837960 (LD of $\mathrm{r}^{2}>0.8$ ), are functional in modulating BACE2 expression. This evidence includes the observation that (i) rs2837960 resides within a haplotypic block that spans

Table 6 Analysis of Top RA-SNPs for Association with AD in Combined Stage 1 and Age-Matched Stage 2

\begin{tabular}{cccccccccc}
\hline & \multicolumn{3}{c}{ Uncorrected Logistic Regression } & \multicolumn{4}{c}{ Regression Corrected for Age, Sex and APOE } \\
CHR & SNP & OR & L95 & U95 & P & OR & L95 & U95 & P \\
\hline $\mathbf{2 1}$ & rs2837960 & 1.239 & 1.083 & 1.418 & 0.002 & 1.199 & 1.032 & 1.392 & 0.018 \\
$\mathbf{1 8}$ & rs2002842 & 1.056 & 0.948 & 1.178 & 0.323 & 1.050 & 0.931 & 1.184 & 0.430 \\
$\mathbf{9}$ & rs3761847 & 1.090 & 0.981 & 1.210 & 0.108 & 1.075 & 0.958 & 1.207 & 0.217 \\
\hline
\end{tabular}

Combined analysis of Stage 1 and Stage 2 data from individuals between 60 and 80 years of age was performed to clarify the association of rs 2837960 ,

rs3761847 and rs2002842 with AD risk. The only SNP found to retain a significant association with AD was rs2837960, the G allele of which appears to increase $\mathrm{AD}$ risk. 


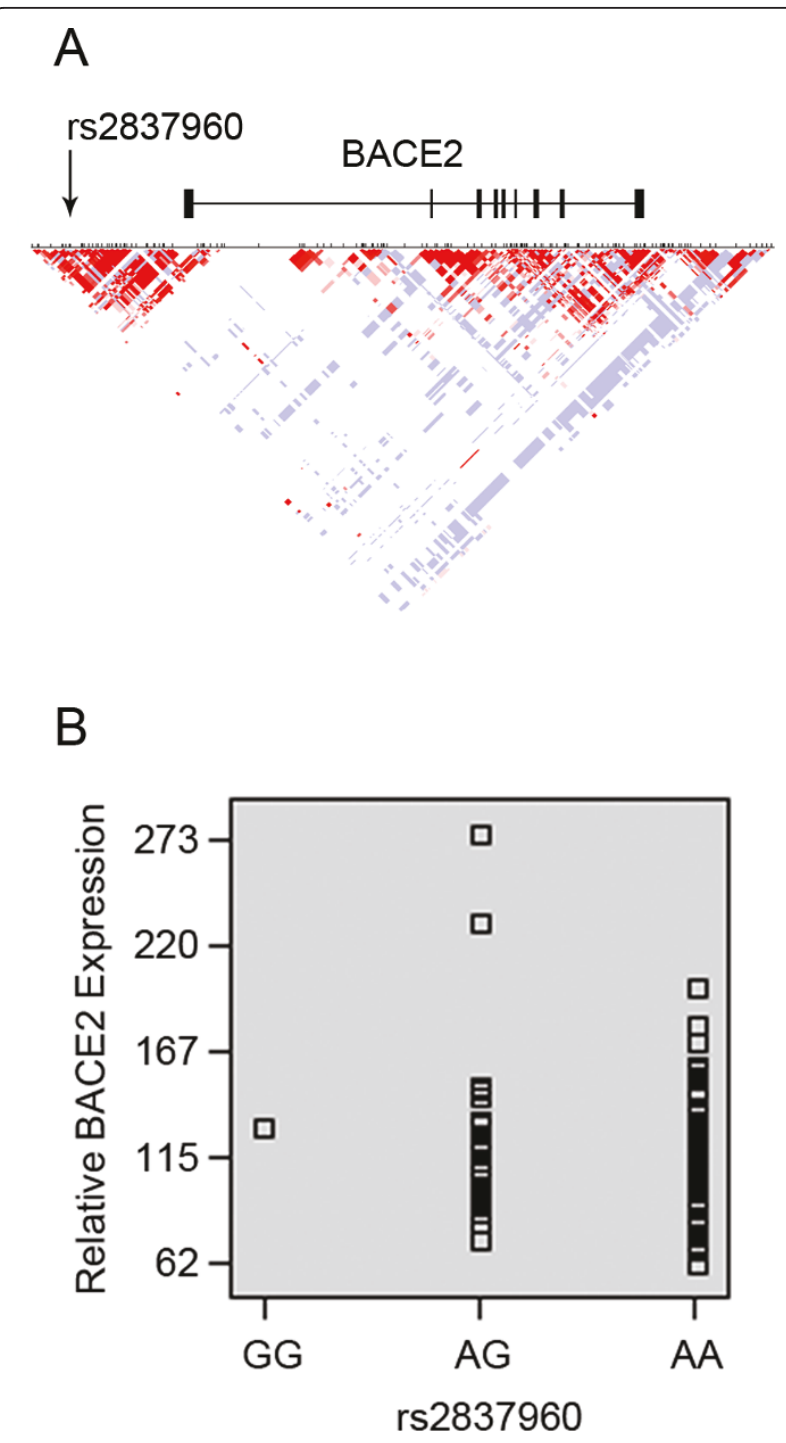

Figure 1 Location of s2837960 and trend between rs2837960 and BACE2 expression. The gene nearest to rs2837960 is BACE2, whose transcription start site is $\sim 27.8 \mathrm{~kb}$ downstream. (A) Analysis of the HapMap CEU population reveals that rs2837960 resides within a linkage disequilibrium block that includes the BACE2 promoter region and first exon. (B) Analysis of exon tiling array data within the SNPExpress database suggests a trend between rs3837960_G and increased BACE2 expression ( $p=0.08$ ).

the region containing both the proximal and distal $B A C E 2$ promoters as well as the 5'UTR and first exon of $B A C E 2$, (ii) the region surrounding rs2837960 and its proxy SNPs $\left(\mathrm{r}^{2}=1.0, \sim 4 \mathrm{~kb}\right.$ window) is well conserved in primates per rVISTA analysis (data not shown), and (iii) the alleles of rs2837960 and its proxy SNPs are predicted to differentially affect transcription factor binding per PROMO 3.0 analysis of the TRANSFAC database (data not shown) [45-47].

Other studies that examined the association between $B A C E 2$ polymorphisms and $\mathrm{AD}$ risk have yielded mixed results [48-54]. These studies differ with our study in that (i) they have focused on SNPs much more proximal to $B A C E 2$ that are not in strong linkage disequilibrium with rs2837960 and (ii) they generally utilized smaller populations than those utilized in our present study. Future analyses of the association between $B A C E 2$ SNPs and AD should therefore take into account SNPs that are more distal to $B A C E 2$, such as rs 2837960 , as well as utilize larger population sizes that are sufficiently powered to detect associations with AD. Thus, in future studies rs2837960 may emerge as a risk factor for both RA and $\mathrm{AD}$ that functionally modulates $B A C E 2$ expression. Elucidation of the precise mechanism by which rs 2837960 , or a SNP that is proxy to it, modulates BACE2 expression may contribute to a better understanding of the role of $B A C E 2$ in both AD and RA pathology.

\section{Conclusions}

In summary, we have provided evidence that RA genetics do not underlie the inverse relationship between RA and $\mathrm{AD}$ but rather may promote $\mathrm{AD}$. Furthermore, we have found that rs2837960 is associated with both RA and AD and that it, or one of its proxy SNPs, may modulate the expression of $B A C E 2$. As we learn more about the pathologic processes behind both RA and $A D$, including the contribution of $B A C E 2$ to each disease, a greater understanding of the factors underlying the inverse relationship between these two diseases may be obtained.

\section{Materials and methods SNP Selection}

The Human Genome (HuGE) Navigator (http://www. hugenavigator.net) was queried using the search term "rheumatoid arthritis" to identify RA-associated SNPs of genome-wide significance [55]. Six available studies utilizing individuals of European decent were chosen to mimic the AD MAYO GWAS demographics (Table 7). Sample sizes ranged from $\sim 1,600$ (810 RA, 794 non-RA) to $\sim 25,500$ (7,322 RA, 18,262 non-RA). Thus, we identified twenty-eight candidate SNPs for study from the literature. SNPs which appeared more than once or that were in tight linkage disequilibrium with each other, i.e. $\mathrm{r}^{2}>0.8$ (according to the CEU HapMap population), were considered to be redundant and only those with the lowest RA-associated p-value were retained for further analysis [17]. This effort reduced the number of candidate SNPs to twenty-two. If a candidate RA-associated SNP was not available within the Mayo Clinic AD GWAS, an appropriate proxy SNP (LD of $\mathrm{r}^{2}>0.8$ ) was selected by using the HapMap-based SNAP proxy search (http://www.broadinstitute.org/mpg/snap/) [56]. Ultimately, seventeen of the candidate SNPs or their proxies were present in our AD GWAS dataset. 


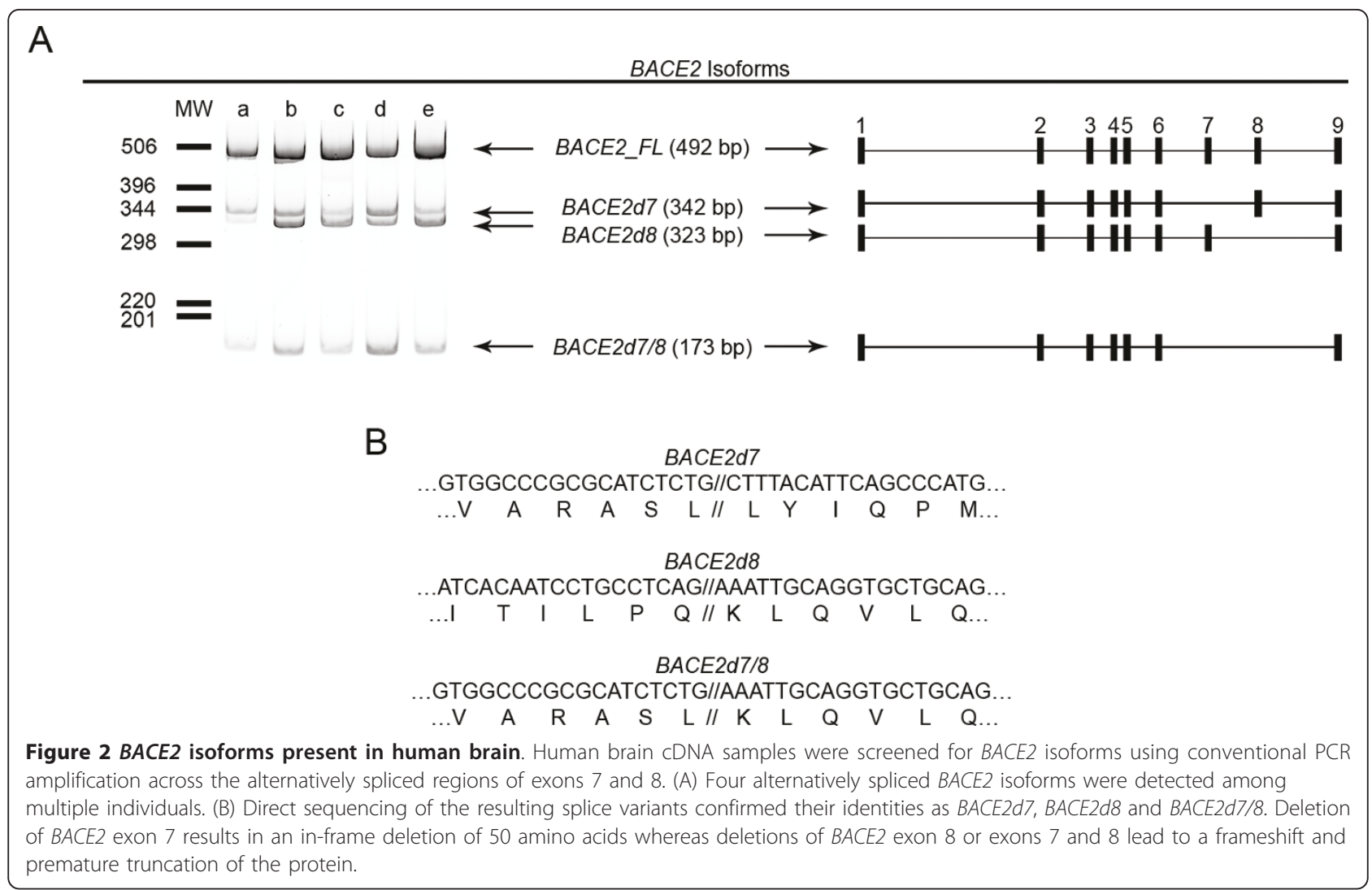

\section{Case and Control Samples}

The Mayo Clinic case-control samples used for the Stage 1 analysis have been described in detail in a prior GWAS publication [57]. The Mayo Clinic case-control series used for the Stage 2 study have also been previously described [58]. Briefly, clinical diagnoses of probable $\mathrm{AD}$ were made according to NINCDS-ADRDA criteria for samples from Jacksonville, FL (JS) and Rochester, MN (RS); age-matched controls had a score of 0 on the Clinical Dementia Rating scale. Additional

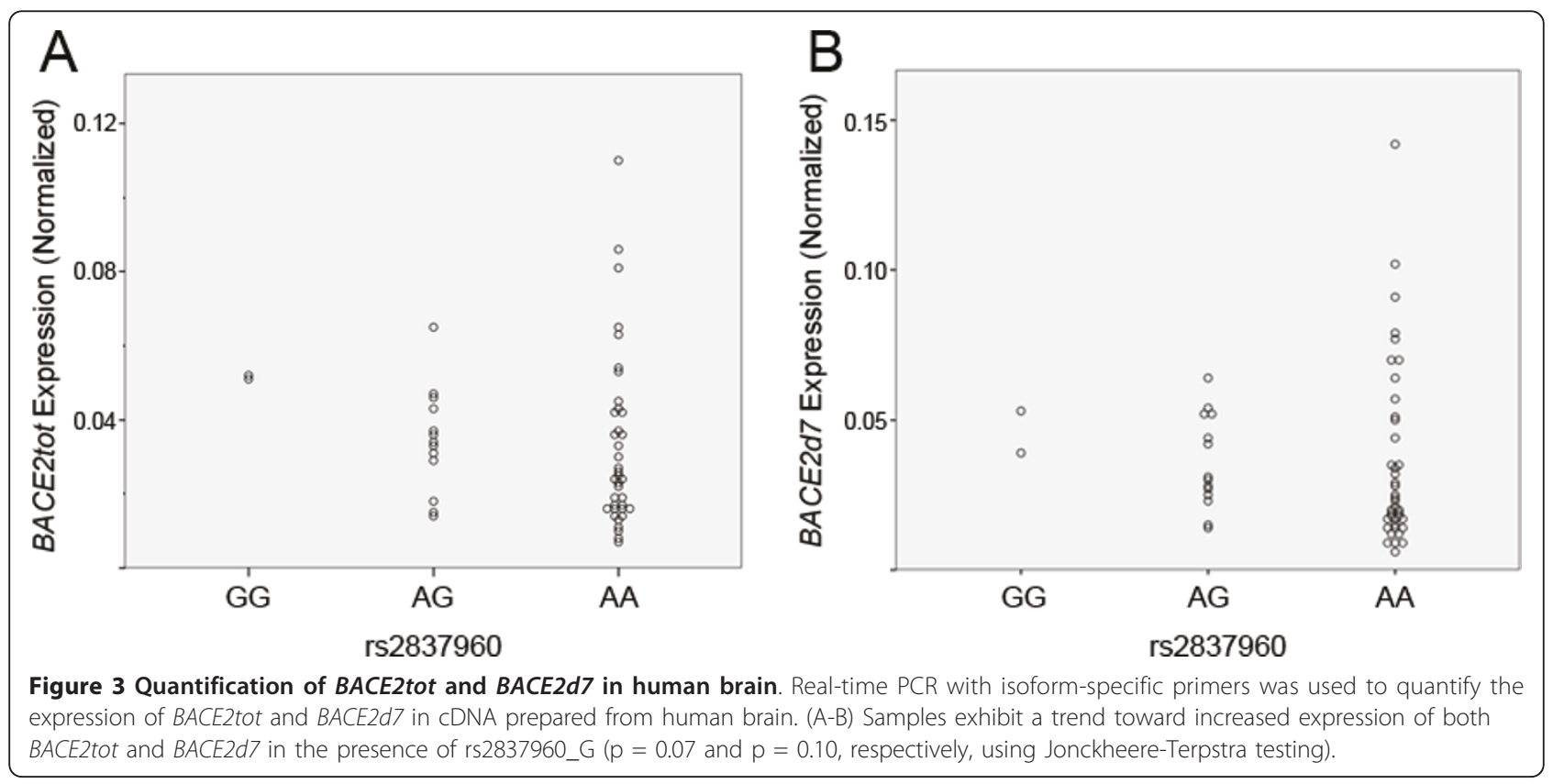


Table 7 RA GWAS reports identifying RA genetic risk factors

\begin{tabular}{ccc}
\hline Article & PMID & \# of GWAS Hits \\
\hline Gregersen et al., Nat Genet, 2009 & 19503088 & 5 \\
Raychaudhuri et al., Nat Genet, 2008 & 18794853 & 9 \\
Julia et al., Arthritis Rheum, 2008 & 18668548 & 2 \\
WTCCC, Nature, 2007 & 17554300 & 7 \\
Plenge et al., N Engl J Med, 2007 & 17804836 & 3 \\
Plenge et al., Nat Genet, 2007 & 17982456 & 2 \\
\hline
\end{tabular}

Six RA GWAS manuscripts were identified by querying the HuGE Navigator database for "rheumatoid arthritis." Together, these studies document twentyeight SNPs that are significantly associated with RA, $p<10^{-6}$. Of these twentyeight SNPs, seventeen (or their proxies) were present in our AD GWAS and evaluated further for their association with AD.

samples were obtained from the Mayo Clinic brain bank (AUT); autopsy-confirmed diagnosis of AD (NINCDSADRDA, Braak score >4.0) was utilized for AD samples while non-AD samples exhibited limited AD pathology (Braak <2.5, not including other unrelated pathology).

\section{AD Association Testing}

Association testing of RA-associated SNPs for AD risk was carried out in two stages by using PLINK software (http://pngu.mgh.harvard.edu/purcell/plink/) [59]. All genotyped samples were subject to strict quality control including elimination of samples with call rates $<90 \%$, MAF $<0.01$, HW $\mathrm{p}<0.001$, discrepancy between reported and genotyped sex, cryptic relatedness and discordant genotype clustering. Stage 1 consisted of 1264 non-AD and $843 \mathrm{AD}$ subjects with average ages of 74.3 \pm 4.5 (age at last assessment, mean \pm SD) and $72.4 \pm$ 4.6 years (age at diagnosis), respectively. The non-AD and $\mathrm{AD}$ groups in this series consisted of $51.7 \%$ and $57.5 \%$ female individuals, respectively. Stage 1 samples were genotyped by using HumanHap300-Duo Genotyping BeadChips processed with an Illumina BeadLab station (Illumina, San Diego, CA) at the Mayo Clinic Genotyping Shared Resource center (Rochester, MN).

We proceeded to test for an association between the seventeen RA-associated SNPs and AD in this Stage 1 case-control population. Stage 1 association testing was performed by using PLINK to generate allelic models that included odds ratios (OR), 95\% confidence intervals $(\mathrm{CI}) \mathrm{s}$ and uncorrected p-values. Logistic regression was also performed using the covariates age, sex and $A P O E$ genotype. With regards to multiple testing we expected to obtain approximately one false positive result given $\alpha$ $=0.05$ (seventeen unique SNPs; 17 tests $\times 0.05=0.85$ ). Bonferroni correction for multiple testing was also applied to data generated using allelic models.

Stage 2 samples were genotyped by using SEQUENOM MassARRAY iPLEX Platform (Sequenom, San Diego, CA). Overall, Stage 2 consisted of 2677 non-AD and $1102 \mathrm{AD}$ subjects with average ages of $81.0 \pm 6.2$ and $83.5 \pm 6.6$ years of age, respectively. The non-AD and $\mathrm{AD}$ groups were composed of $55.0 \%$ and $64.0 \%$ female individuals, respectively. Stage 2 AD-SNP association testing was performed using only the three SNPs identified in Stage 1 as being associated with both RA and AD. PLINK software was used to generate odds ratios, 95\% CIs and p-values per allelic modeling. Logistic regression including the covariates age, sex and $A P O E$ genotype was also performed. To evaluate the overall significance of Stage 1 and 2 data, they were combined and examined collectively.

Due to the considerable difference in mean age between Stage 1 and Stage 2 individuals, and due to our interest in focusing on genetic, rather than environmental factors, we also chose to examine only Stage 2 individuals between 60 and 80 years of age. Hence, when Stage 2 was limited to individuals between 60 and 80 years of age, our analysis included 912 non-AD and 186 AD subjects with average ages of $73.9 \pm 3.8$ and $72.8 \pm$ 5.1 years. The non-AD and AD groups consisted of $49.9 \%$ and $57.0 \%$ female individuals, respectively. Similar to our analysis of our initial Stage 2 population, logistic regression of this modified Stage 2 population was also performed to test for an association between the three AD-associated SNPs from Stage 1. Furthermore, we evaluated the overall significance of RA-associated SNP associations with AD in combined Stage 1 and Stage 2 individuals between 60 and 80 years of age.

\section{Human Tissue}

Human anterior cingulate brain specimens were generously provided by the Sanders-Brown AD Center Neuropathology Core and have been described elsewhere [60]. The samples were from deceased individuals with an average age at death for females of $82 \pm 7$ years (mean $\pm \mathrm{SD}, \mathrm{n}=29)$ and for males of $81 \pm 8(\mathrm{n}=24)$. The average postmortem interval (PMI) for females and males was $3.2 \pm 0.8 \mathrm{~h}$ and $3.0 \pm 0.8 \mathrm{~h}$, respectively.

\section{Evaluation of BACE2 isoforms in vivo}

To gain insights into the functionality of rs 2837960 we tested for an association between rs2837960 and BACE2 expression in human brain. We first screened anterior cingulate samples for the presence of $B A C E 2$ and its known alternatively spliced isoforms that lack exons 7 and 8, respectively. Total RNA and genomic DNA were prepared from human tissue samples; the RNA was reverse transcribed as we have reported elsewhere $[61,62]$. Conventional PCR using Platinum Taq (Invitrogen, Carlsbad, CA) was used to amplify the region of BACE2 spanning exons 6-9 (Table 8). Thermal cycling conditions consisted of denaturation at $95^{\circ} \mathrm{C}$ for $5 \mathrm{~min}$ followed by 32 cycles of $95^{\circ} \mathrm{C}$ for $30 \mathrm{~s}, 60^{\circ} \mathrm{C}$ for $30 \mathrm{~s}$, $72^{\circ} \mathrm{C}$ for $1 \mathrm{~min}$ and a final extension at $72^{\circ} \mathrm{C}$ for $2 \mathrm{~min}$. 
Table 8 Primers used for analyses of BACE2 isoform expression

\begin{tabular}{ll}
\hline BACE2 Isoform screening primers \\
\hline BACE2 Exon 6 Forward: & ATAACGCAGACAAGGCCATC \\
\hline BACE2 Exon 9 Reverse: & GGACACAGTTGCTGGCTACA \\
\hline BACE2 isoform specific RT-PCR primers & \\
\hline BACE2 Exon 6 Forward: & GCCCCAGAAGGTGTTGAT \\
\hline BACE2 Exon 6-8 Junction Reverse: & GGCTGAATGTAAAGCAGAG \\
\hline BACE2 Exon 5 Forward: & TGGGTGAATTGACCAAGT \\
\hline BACE2 Exon 6 Reverse: & GATGGCCTTGTCTGCGTAT \\
\hline
\end{tabular}

PCR products were separated using $8 \%$ TBE-PAGE gel electrophoresis and visualized using SYBR-gold fluorescent stain (Invitrogen) and a fluorescence imager (FLA2000, Fuji). To confirm the identities of the BACE2 splice variants, bands were excised, purified and directly sequenced (Davis Sequencing, Davis, CA).

To quantify total $B A C E 2$ expression (BACE2tot) and expression of the $B A C E 2$ isoform lacking exon 7 $(B A C E 2 d 7)$ we designed separate primer sets. BACE2tot expression was measured by amplification of a product spanning a non-alternatively spliced region of $B A C E 2$ (exons 5-6). Isoform-specific primers designed to amplify $B A C E 2 d 7$ consisted of a forward primer specific to $B A C E 2$ exon 6 and a reverse primer specific to the junction of exons 6-8 (Table 8). DNA samples were genotyped using a TaqMan SNP Genotyping Assay (ID \# C_2688271_10; ABI, Carlsbad, CA).

Quantitative real-time PCR reactions contained $\sim 20$ ng of sample cDNA together with $10 \mu \mathrm{l}$ of PerfeCTA SYBR green SuperMix (Quanta Biosciences, Gaithersburg, MD), $10 \mu \mathrm{l}$ of ddH2O and $20 \mathrm{pmol}$ of forward and reverse primers. Cycling conditions included a 3 minute denaturation step at $95^{\circ} \mathrm{C}$ followed by 40 cycles of denaturation for 15 seconds at $95^{\circ} \mathrm{C}$ and annealing/ extension for 45 seconds at $60^{\circ} \mathrm{C}$ using an MJ Opticon 4 thermal cycler (Biorad, Hercules, CA). A melting curve was generated following cycling to assess the purity of amplification product. Fidelity of amplification was also assessed via visual inspection of PCR products on $8 \%$ TBE-PAGE gel stained with SYBR gold. Standard curves were generated from purified PCR products that were quantified by A260/280 spectrophotometric analysis. Standard curves were then used to calculate the copy number for each $B A C E 2$ isoform measured.

Hypoxanthine-guanine phosphoribosyltransferase (HPRT) and ribosomal protein L32 (RPL32) were used as housekeeping genes per the analysis of geNorm software as described previously [63-65]. Expression levels of each of these genes were measured by using quantitative real-time PCR and gene specific primers under conditions identical to cycling conditions for $B A C E 2$.
Standard curves were used to generate exact copy numbers, which in turn were used to calculate the samplespecific geometric mean of HPRT and RPL32 expression. The geometric mean was in turn used to normalize subsequent $B A C E 2$ expression data. Analysis of the association between $B A C E 2$ isoforms and rs2837960 genotype was performed using non-parametric Jonckheere-Terpstra testing (PASW Statistics, v.18, IBM, Somers, NY).

\section{Acknowledgements and Funding}

This work was supported by grants from the National Institutes of Health/ National Institute on Aging P01-AG030128 (SE), RO1-AG18023 (SGY), T32AG000242 (CRS) and Mayo Alzheimer's Disease Research Center P50-

AG16574 (SGY). Human tissue was generously provided by the University of Kentucky AD Center, which is supported by P30-AG028383.

\section{Author details}

${ }^{1}$ Department of Physiology, Sanders-Brown Center on Aging, University of Kentucky, Lexington, KY, USA. ${ }^{2}$ Department of Neuroscience, Mayo Clinic College of Medicine, Jacksonville, FL, USA.

\section{Authors' contributions}

CRS and SE contributed to experimental design and wrote the manuscript CRS performed RT-PCR of BACE2 isoforms, rs2837960 genotyping of human brain tissue and association testing between BACE2 isoform expression and rs2837960. FZ performed SEQUENOM genotyping of samples for Stage 2 analyses. SGY, FZ and CRS performed statistical analysis of RA-associated SNPs and AD GWAS data. All authors have read and approved the final manuscript.

\section{Competing interests}

The authors declare that they have no competing interests.

Received: 25 February 2011 Accepted: 19 May 2011

Published: 19 May 2011

\section{References}

1. Jenkinson ML, Bliss MR, Brain AT, Scott DL: Rheumatoid arthritis and senile dementia of the Alzheimer's type. Br J Rheumatol 1989, 28:86-88.

2. McGeer PL, McGeer E, Rogers J, Sibley J: Anti-inflammatory drugs and Alzheimer disease. Lancet 1990, 335:1037.

3. Myllykangas-Luosujarvi $\mathrm{R}$, Isomaki H: Alzheimer's disease and rheumatoid arthritis. Br J Rheumatol 1994, 33:501-502.

4. Scott DL, Wolfe F, Huizinga TW: Rheumatoid arthritis. Lancet 2010, 376:1094-1108

5. Gatz M, Reynolds CA, Fratiglioni L, Johansson B, Mortimer JA, Berg S, Fiske A, Pedersen NL: Role of genes and environments for explaining Alzheimer disease. Arch Gen Psychiatry 2006, 63:168-174.

6. Weggen S, Eriksen JL, Das P, Sagi SA, Wang R, Pietrzik CU, Findlay KA, Smith TE, Murphy MP, Bulter T, et al: A subset of NSAIDs lower amyloidogenic Abeta42 independently of cyclooxygenase activity. Nature 2001, 414:212-216.

7. Eriksen $J$, Sagi SA, Smith TE, Weggen S, Das P, McLendon DC, Ozols W, Jessing KW, Zavitz KH, Koo EH, Golde TE: NSAIDs and enantiomers of flurbiprofen target gamma-secretase and lower Abeta 42 in vivo. J Clin Invest 2003, 112:440-449.

8. Rogers J, Kirby LC, Hempelman SR, Berry DL, McGeer PL, Kaszniak AW, Zalinski J, Cofield M, Mansukhani L, Willson P, et al: Clinical trial of indomethacin in Alzheimer's disease. Neurology 1993, 43:1609-1611.

9. Breitner JC, Gau BA, Welsh KA, Plassman BL, McDonald WM, Helms MJ, Anthony JC: Inverse association of anti-inflammatory treatments and Alzheimer's disease: initial results of a co-twin control study. Neurology 1994, 44:227-232.

10. Szekely CA, Green RC, Breitner JC, Ostbye T, Beiser AS, Corrada MM, Dodge $\mathrm{HH}$, Ganguli $\mathrm{M}$, Kawas $\mathrm{CH}$, Kuller $\mathrm{LH}$, et al: No advantage of $\mathrm{A}$ beta 42-lowering NSAIDs for prevention of Alzheimer dementia in six pooled cohort studies. Neurology 2008, 70:2291-2298. 
11. Szekely CA, Breitner JC, Fitzpatrick AL, Rea TD, Psaty BM, Kuller LH, Zandi PP: NSAID use and dementia risk in the Cardiovascular Health Study: role of APOE and NSAID type. Neurology 2008, 70:17-24.

12. Breitner JC, Haneuse SJ, Walker R, Dublin S, Crane PK, Gray SL, Larson EB: Risk of dementia and AD with prior exposure to NSAIDs in an elderly community-based cohort. Neurology 2009, 72:1899-1905.

13. Sonnen JA, Larson EB, Walker RL, Haneuse S, Crane PK, Gray SL, Breitner JC, Montine TJ: Nonsteroidal anti-inflammatory drugs are associated with increased neuritic plaques. Neurology 2010, 75:1203-1210.

14. Ahmed RR, Holler CJ, Webb RL, Li F, Beckett TL, Murphy MP: BACE1 and BACE2 enzymatic activities in Alzheimer's disease. J Neurochem 2010, 112:1045-1053.

15. Bettegazzi B, Mihailovich M, Di Cesare A, Consonni A, Macco R, Pelizzoni I, Codazzi F, Grohovaz F, Zacchetti D: beta-Secretase activity in rat astrocytes: translational block of BACE1 and modulation of BACE2 expression. The European journal of neuroscience 2011, 33:236-243.

16. Farrer LA, Cupples LA, Haines JL, Hyman B, Kukull WA, Mayeux R, Myers RH, Pericak-Vance MA, Risch N, van Duijn CM: Effects of age, sex, and ethnicity on the association between apolipoprotein $\mathrm{E}$ genotype and Alzheimer disease. A meta-analysis. APOE and Alzheimer Disease Meta Analysis Consortium. JAMA 1997, 278:1349-1356.

17. Frazer KA, Ballinger DG, Cox DR, Hinds DA, Stuve LL, Gibbs RA, Belmont JW, Boudreau A, Hardenbol P, Leal SM, et al: A second generation human haplotype map of over 3.1 million SNPs. Nature 2007, 449:851-861.

18. Lahiri DK, Maloney B, Ge YW: Functional domains of the BACE1 and BACE2 promoters and mechanisms of transcriptional suppression of the BACE2 promoter in normal neuronal cells. J Mol Neurosci 2006, 29:65-80.

19. Maloney B, Ge YW, Greig NH, Lahiri DK: Characterization of the human beta-secretase 2 (BACE2) 5'-flanking region: identification of a 268-bp region as the basal BACE2 promoter. J Mol Neurosci 2006, 29:81-99.

20. Sun X, Wang Y, Qing H, Christensen MA, Liu Y, Zhou W, Tong Y, Xiao C, Huang $Y$, Zhang $S$, et al: Distinct transcriptional regulation and function of the human BACE2 and BACE1 genes. Faseb J 2005, 19:739-749.

21. Sanders MA, Verhaak RG, Geertsma-Kleinekoort WM, Abbas S, Horsman S, van der Spek PJ, Lowenberg B, Valk PJ: SNPExpress: integrated visualization of genome-wide genotypes, copy numbers and gene expression levels. BMC Genomics 2008, 9:41.

22. Farzan M, Schnitzler CE, Vasilieva N, Leung D, Choe H: BACE2, a betasecretase homolog, cleaves at the beta site and within the amyloid-beta region of the amyloid-beta precursor protein. Proc Natl Acad Sci USA 2000, 97:9712-9717.

23. Stockley JH, Ravid R, O'Neill C: Altered beta-secretase enzyme kinetics and levels of both BACE1 and BACE2 in the Alzheimer's disease brain. FEBS Lett 2006, 580:6550-6560.

24. Sloan CD, Shen L, West JD, Wishart HA, Flashman LA, Rabin LA, Santulli RB, Guerin SJ, Rhodes CH, Tsongalis GJ, et al: Genetic pathway-based hierarchical clustering analysis of older adults with cognitive complaints and amnestic mild cognitive impairment using clinical and neuroimaging phenotypes. Am J Med Genet B Neuropsychiatr Genet 2010, 153B:1060-1069.

25. Amor S, Puentes F, Baker D, van der Valk P: Inflammation in neurodegenerative diseases. Immunology 2010, 129:154-169.

26. Perry VH, Nicoll JA, Holmes C: Microglia in neurodegenerative disease. Nat Rev Neurol 2010, 6:193-201.

27. Metcalfe MJ, Figueiredo-Pereira ME: Relationship between tau pathology and neuroinflammation in Alzheimer's disease. Mt Sinai J Med 2010, 77:50-58.

28. Bertram L, McQueen MB, Mullin K, Blacker D, Tanzi RE: Systematic metaanalyses of Alzheimer disease genetic association studies: the AlzGene database. Nat Genet 2007, 39:17-23.

29. Xie Z, Harris-White ME, Wals PA, Frautschy SA, Finch CE, Morgan TE: Apolipoprotein J (clusterin) activates rodent microglia in vivo and in vitro. J Neurochem 2005, 93:1038-1046.

30. Seshadri S, Fitzpatrick AL, Ikram MA, DeStefano AL, Gudnason V, Boada M, Bis JC, Smith AV, Carassquillo MM, Lambert JC, et al: Genome-wide analysis of genetic loci associated with Alzheimer disease. JAMA 2010, 303:1832-1840.

31. Zanjani H, Finch CE, Kemper C, Atkinson J, McKeel D, Morris JC, Price JL: Complement activation in very early Alzheimer disease. Alzheimer Dis Assoc Disord 2005, 19:55-66.
32. McGeer EG, McGeer PL: Neuroinflammation in Alzheimer's disease and mild cognitive impairment: a field in its infancy. J Alzheimers Dis 2010, 19:355-361.

33. McCoy MK, Tansey MG: TNF signaling inhibition in the CNS: implications for normal brain function and neurodegenerative disease. J Neuroinflammation 2008, 5:45.

34. Conductier G, Blondeau N, Guyon A, Nahon JL, Rovere C: The role of monocyte chemoattractant protein MCP1/CCL2 in neuroinflammatory diseases. J Neuroimmunol 2010

35. El Khoury J, Toft M, Hickman SE, Means TK, Terada K, Geula C, Luster AD: Ccr2 deficiency impairs microglial accumulation and accelerates progression of Alzheimer-like disease. Nat Med 2007, 13:432-438.

36. Britschgi M, Wyss-Coray T: Immune cells may fend off Alzheimer disease. Nat Med 2007, 13:408-409.

37. Heinzen EL, Ge D, Cronin KD, Maia JM, Shianna KV, Gabriel WN, WelshBohmer KA, Hulette CM, Denny TN, Goldstein DB: Tissue-specific genetic control of splicing: implications for the study of complex traits. PLOS Biol 2008, 6:e1

38. Akiyama H, Barger S, Barnum S, Bradt B, Bauer J, Cole GM, Cooper NR, Eikelenboom P, Emmerling M, Fiebich BL, et al: Inflammation and Alzheimer's disease. Neurobiol Aging 2000, 21:383-421.

39. Aisen PS: Anti-inflammatory therapy for Alzheimer's disease. Neurobiol Aging 2000, 21:447-448, discussion 451-443.

40. Zandi PP, Anthony JC, Hayden KM, Mehta K, Mayer L, Breitner JC: Reduced incidence of $A D$ with NSAID but not $\mathrm{H} 2$ receptor antagonists: the Cache County Study. Neurology 2002, 59:880-886.

41. Aisen PS, Schafer KA, Grundman M, Pfeiffer E, Sano M, Davis KL, Farlow MR, Jin S, Thomas RG, Thal LJ: Effects of rofecoxib or naproxen vs placebo on Alzheimer disease progression: a randomized controlled trial. JAMA 2003, 289:2819-2826

42. de Jong D, Jansen R, Hoefnagels W, Jellesma-Eggenkamp M, Verbeek M, Borm G, Kremer B: No effect of one-year treatment with indomethacin on Alzheimer's disease progression: a randomized controlled trial. PLOS One 2008, 3:e1475.

43. Solans A, Estivill X, de La Luna S: A new aspartyl protease on 21q22.3, BACE2, is highly similar to Alzheimer's amyloid precursor protein betasecretase. Cytogenet Cell Genet 2000, 89:177-184.

44. Dominguez D, Tournoy J, Hartmann D, Huth T, Cryns K, Deforce S, Serneels L, Camacho IE, Marjaux E, Craessaerts K, et al: Phenotypic and biochemical analyses of BACE1- and BACE2-deficient mice. J Biol Chem 2005, 280:30797-30806

45. Frazer KA, Pachter L, Poliakov A, Rubin EM, Dubchak I: VISTA: computational tools for comparative genomics. Nucleic Acids Res 2004, 32: W273-279

46. Messeguer X, Escudero R, Farre D, Nunez O, Martinez J, Alba MM: PROMO: detection of known transcription regulatory elements using speciestailored searches. Bioinformatics 2002, 18:333-334.

47. Farre D, Roset R, Huerta M, Adsuara JE, Rosello L, Alba MM, Messeguer X: Identification of patterns in biological sequences at the ALGGEN server: PROMO and MALGEN. Nucleic Acids Res 2003, 31:3651-3653.

48. Giedraitis V, Kilander L, Degerman-Gunnarsson M, Sundelof J, Axelsson T, Syvanen AC, Lannfelt L, Glaser A: Genetic analysis of Alzheimer's disease in the Uppsala Longitudinal Study of Adult Men. Dementia and geriatric cognitive disorders 2009, 27:59-68.

49. Gold G, Blouin JL, Herrmann FR, Michon A, Mulligan R, Duriaux Sail G, Bouras C, Giannakopoulos P, Antonarakis SE: Specific BACE1 genotypes provide additional risk for late-onset Alzheimer disease in APOE epsilon 4 carriers. American journal of medical genetics Part B, Neuropsychiatric genetics: the official publication of the International Society of Psychiatric Genetics 2003, 119B:44-47.

50. Li H, Wetten S, Li L, St Jean PL, Upmanyu R, Surh L, Hosford D, Barnes MR, Briley JD, Borrie $M$, et al: Candidate single-nucleotide polymorphisms from a genomewide association study of Alzheimer disease. Archives of neurology 2008, 65:45-53.

51. Myllykangas L, Wavrant-De Vrieze F, Polvikoski T, Notkola IL, Sulkava R, Niinisto L, Edland SD, Arepalli S, Adighibe O, Compton D, et al: Chromosome 21 BACE2 haplotype associates with Alzheimer's disease: a two-stage study. J Neurol Sci 2005, 236:17-24.

52. Nowotny P, Kwon JM, Chakraverty S, Nowotny V, Morris JC, Goate AM: Association studies using novel polymorphisms in BACE1 and BACE2. Neuroreport 2001, 12:1799-1802. 
53. Reiman EM, Webster JA, Myers AJ, Hardy J, Dunckley T, Zismann VL, Joshipura KD, Pearson JV, Hu-Lince D, Huentelman MJ, et al: GAB2 alleles modify Alzheimer's risk in APOE epsilon4 carriers. Neuron 2007, 54:713-720.

54. Yu Y, Jia J: Lack of association between the polymorphisms of beta-site APP-cleaving enzyme 2 (BACE2) 5'-flanking region and sporadic Alzheimer's disease. Brain Res 2009, 1257:10-15.

55. Yu W, Gwinn M, Clyne M, Yesupriya A, Khoury MJ: A navigator for human genome epidemiology. Nature genetics 2008, 40:124-125.

56. Johnson AD, Handsaker RE, Pulit SL, Nizzari MM, O'Donnell CJ, de Bakker PI: SNAP: a web-based tool for identification and annotation of proxy SNPs using HapMap. Bioinformatics 2008, 24:2938-2939.

57. Carrasquillo MM, Zou F, Pankratz VS, Wilcox SL, Ma L, Walker LP, Younkin SG, Younkin CS, Younkin LH, Bisceglio GD, et al: Genetic variation in PCDH11X is associated with susceptibility to late-onset Alzheimer's disease. Nat Genet 2009, 41:192-198.

58. Carrasquillo MM, Belbin O, Hunter TA, Ma L, Bisceglio GD, Zou F, Crook JE, Pankratz VS, Dickson DW, Graff-Radford NR, et al: Replication of CLU, CR1, and PICALM associations with alzheimer disease. Arch Neurol 2010, 67:961-964.

59. Purcell S, Neale B, Todd-Brown K, Thomas L, Ferreira MA, Bender D, Maller J, Sklar P, de Bakker PI, Daly MJ, Sham PC: PLINK: a tool set for wholegenome association and population-based linkage analyses. Am J Hum Genet 2007, 81:559-575.

60. Zou F, Gopalraj RK, Lok J, Zhu H, Ling IF, Simpson JF, Tucker HM, Kelly JF, Younkin SG, Dickson DW, et al: Sex-dependent association of a common low-density lipoprotein receptor polymorphism with RNA splicing efficiency in the brain and Alzheimer's disease. Hum Mol Genet 2008, 17:929-935.

61. Zhu H, Tucker HM, Grear KE, Simpson JF, Manning AK, Cupples LA, Estus S: A common polymorphism decreases low-density lipoprotein receptor exon 12 splicing efficiency and associates with increased cholesterol. Hum Mol Genet 2007, 16:1765-1772.

62. Ling IF, Gopalraj RK, Simpson JF, Estus S: Expression and regulation of a low-density lipoprotein receptor exon 12 splice variant. J Neurochem 2010.

63. Vandesompele J, De Preter K, Pattyn F, Poppe B, Van Roy N, De Paepe A, Speleman F: Accurate normalization of real-time quantitative RT-PCR data by geometric averaging of multiple internal control genes. Genome Biol 2002, 3:RESEARCH0034.

64. Ling IF, Estus S: Role of SFRS13A in low-density lipoprotein receptor splicing. Hum Mutat 2010, 31:702-709.

65. Zhang $X$, Ding L, Sandford AJ: Selection of reference genes for gene expression studies in human neutrophils by real-time PCR. BMC Mol Biol 2005, 6:4.

doi:10.1186/1750-1326-6-33

Cite this article as: Simmons et al:: Rheumatoid arthritis-associated polymorphisms are not protective against Alzheimer's disease. Molecular Neurodegeneration 2011 6:33.

\section{Submit your next manuscript to BioMed Central and take full advantage of:}

- Convenient online submission

- Thorough peer review

- No space constraints or color figure charges

- Immediate publication on acceptance

- Inclusion in PubMed, CAS, Scopus and Google Scholar

- Research which is freely available for redistribution

Submit your manuscript at www.biomedcentral.com/submit
Biomed Central 